\title{
Modified Kinematic Technique for Measuring Pathological Hyperextension and Hypermobility of the Interphalangeal Joints
}

\author{
Cheryl D. Metcalf*, Member, IEEE, and Scott V. Notley
}

\begin{abstract}
Dynamic finger joint motion is difficult to measure using optical motion analysis techniques due to the limited surface area allowed for adequate marker placement. This paper describes an extension of a previously validated kinematic measurement technique using a reduced surface marker set and outlines the required calculations based on a specific surface marker placement to calculate flexion/extension and hyperextension of the metacarpophalangeal, proximal interphalangeal, and distal interphalangeal joints. The modified technique has been assessed for accuracy using a series of static reference frames (absolute residual error $= \pm 3.7^{\circ}$, cross correlation between new method and reference frames; $r=0.99$ ). The method was then applied to a small group of participants with rheumatoid arthritis (seven females, one male; mean age $=62.8$ years \pm 12.04 ) and illustrated congruent strategies of movement for a participant and a large range of finger joint movement over the sample $\left(5.8-71.1^{\circ}\right.$, smallest to largest active range of motion). This method used alongside the previous paper [1] provides a comprehensive, validated method for calculating 3-D wrist, hand, fingers, and thumb kinematics to date and provides a valuable measurement tool for clinical research.
\end{abstract}

Index Terms-Finger, hand, hyperextension, kinematic.

\section{INTRODUCTION}

$\mathbf{M}$ EASUREMENT of finger joint movement is important in maintaining functional capacity and optimizing ability to adapt quickly to perturbations in manual activities. Measurement of small joint movement is challenging due to the small area, or active range of motion (AROM), over which the measurement takes place. This complexity is compounded when additional characteristics interfere with the normal structure and function of the joint, such as joint deformity due to arthritic conditions or fixed contractures due to neurological impairment. It is also paramount that any measurements that are taken are valid and clinically applicable in order to inform rehabilitation techniques, orthotic, and surgical interventions.

Manuscript received July 6, 2010; revised October 18, 2010 and December 14, 2010; accepted December 27, 2010. Date of publication January 13, 2011 date of current version April 20, 2011. This work was supported by the Life Sciences Interfaces Forum at the University of Southampton, Southampton, U.K. Asterisk indicates corresponding author.

*C. D. Metcalf is with the Faculty of Health Sciences, University of Southampton, Southampton, SO17 1 BJ, U.K. (e-mail: c.d.metcalf@soton. ac.uk).

S. V. Notley was with the Institute of Sound and Vibration Research, University of Southampton, Southampton, SO17 1 BJ, U.K. (e-mail: scott.notley106@googlemail.com).

Color versions of one or more of the figures in this paper are available online at http://ieeexplore.ieee.org.

Digital Object Identifier 10.1109/TBME.2011.2106126

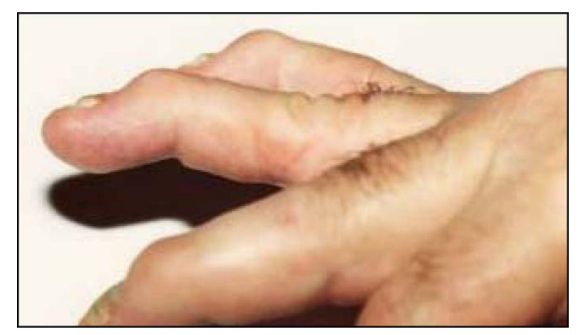

Fig. 1. Swan-neck deformity classified by hyperextension of the PIP joint and flexion of the DIP joint of the finger.

Specifically, in rheumatoid arthritis (RA) a typical symptom is swan-neck deformity, which is classified by hyperextension of the proximal interphalangeal (PIP) joint and flexion of the distal interphalangeal (DIP) joint of the finger [2]. Fig. 1 illustrates the static position of swan-neck deformity. More severe cases of swan-neck deformity are corrected with surgical and various splinting techniques [3], for example thermoplastic Oval-8 splints [4], Murphy splints [5], and, more recently, silver ring splints [6], [7]. Given the complexity of resultant finger positions and AROM with impairment, an accurate method of measurement is required. The purpose of this study was to extend a previously validated kinematic measurement technique [1], defining and validating the mathematical method for measurement finger joint AROM, including pathological motion, and applying the extended technique to a sample of participants with RA as an example clinical application.

\section{Measuring Small Joint Movement}

\section{A. Traditional Clinical Techniques}

In the context of assessing finger joint AROM, traditional clinical techniques have opted to use manual goniometry, which has a reported accuracy of 7-9 $9^{\circ}[8]$. Traditional methods of measuring pathological PIP joint hyperextension are defined by the amount of total active motion [9]. Total active motion (TAM) provides a measure of joint range of motion at the metacarpophalangeal (MCP), PIP, and DIP joints of the finger and is based on static goniometric measurements at the limits of the movement range. The normative range for the PIP joint is defined by the TAM as $+60^{\circ}$ to $-20^{\circ}$, where $0^{\circ}$ at the PIP joint is in a neutral position. However, an inherent problem with using TAM to measure PIP joint motion is that it does not distinguish between loss of extension motion and hyperextension motion. 
For example, an inability to fully extend the finger to its neutral position $\left(0^{\circ}\right)$ by $10^{\circ}$ generates the same outcome using the TAM measurement technique as hyperextension of the PIP joint by $10^{\circ}$. Both measurement outcomes would define range of motion from $+60^{\circ}$ to $-10^{\circ}$, whether that range is due to inability to fully extend the finger joint, or due, in fact, to pathological hyperextension. It should be noted, however, that the range of motion defined by the TAM technique is in contrast with the AROM defined for an unimpaired PIP joint in the general literature [2], [10], [11].

\section{B. Kinematic Measurement Techniques}

Kinematic measurement of finger joint motion is a viable alternative for clinical research; however, it is challenging due to the small surface area and can be compounded by the presence of joint deformity. Current methods of kinematic measurement have concentrated on definitions of kinematic standards [12], calculation of joint centers [13], [14], forces acting through the joints during functional grip [15], range of motion assessment [14], [16]-[19], and as an indicator of pathology when compared to a control group [15], [18], [19]. Various methods of marker topology are also possible and comprise different levels of complexity in terms of placement and physical attributes.

When adopting a kinematic measurement technique, selecting an appropriate marker topology will be dependent on the requirements of the proposed trial protocol. Biomechanical investigations, where the emphasis is on analyzing anatomically accurate joint movement, finger flexion/extension, abduction/adduction, and particularly rotation, have adopted complex marker topologies and placement protocols. For example, Fowler and Nicol [15] use several marker clusters placed on the upper arm, forearm, hand, proximal, medial, and distal phalanges of the index finger. However, these six marker clusters (18 markers in total) are required by the associated measurement technique to calculate movement of the index finger alone and the authors have commented on the protocol only being applicable to one finger at a time. Miyata et al. [14] adopted a technical marker set (three noncollinear markers per phalanx) on the hand at the proximal, medial, and distal phalanges of each finger. Their method was specifically developed to help userfriendly, ergonomic product design and measure finger and wrist movement. Chiu [18] adopted a protocol that uses two markers per phalanx and investigated the AROM of injured fingers. However, these complex marker topologies and placement protocols are often not suitable for clinical-based research trials, particularly those involving joint deformation, nodules, skin abnormalities, or smaller surface areas, such as trials involving children. Often, more complicated marker protocols require more surface area and may interfere with movements the participant would naturally undertake. This may include physically impeding the movement of the joint and/or adjoining fingers, or distract the participant, which is particularly relevant when assessing neurologically impaired participants [21].

To date, one kinematic method calculates finger joint hyperextension, which is often present in neuromusculoskeletal conditions. Chiu et al. [20] investigated impaired finger joint

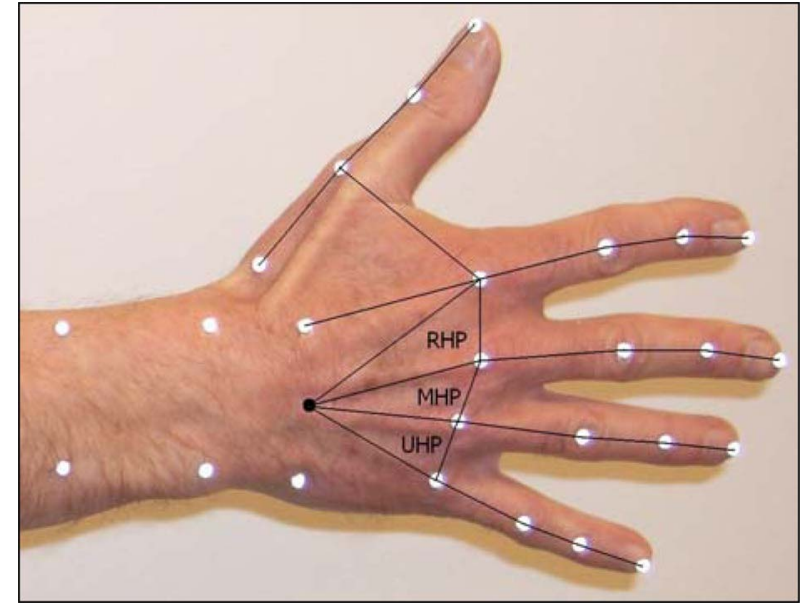

Fig. 2. Marker placement and definitions of planes for the dorsal aspect of the metacarpal arch, where $\mathrm{RHP}=$ radial hand plane, $\mathrm{MHD}=$ middle hand plane, and $\mathrm{UHP}=$ ulnar hand plane.

movements by measuring hyperextension using motion analysis techniques. A group of 12 participants (33 fingers in total) with impairments and injuries to their fingers were included in the trial. Three participants exhibited MCP joint hyperextension of up to $17^{\circ}$ following a misaligned fracture and two crush injuries. Given the limitations of current kinematic measurement techniques in this area, a comprehensive method is required to extend previous techniques [1], [21] based on a reduced marker set specifically to calculate dynamic finger joint hyperextension.

\section{METHOD}

A previously developed kinematic measurement technique that had been tested for accuracy and reliability [1] was extended and used as a basis for calculating finger joint hyperextension. The method was previously tested for validity and reliability (validity $= \pm 1^{\circ}$; interrater reliability of marker placement $= \pm 5^{\circ}$ ). The associated single surface marker topology and placement protocol remained unchanged from the original method, whereby 3-mm hemispherical reflective markers were placed at the forearm and wrist, the first, second, and fifth carpometacarpal (CMC) joints, and each of the MCP, PIP, DIP, and fingertips (see Fig. 2).

The original method was developed to measure joint range of motion and assumed no extreme movements at the joints such as hypermobility and joint hyperextension. Therefore, in order to calculate the joint ranges of movement required by the current objective, the method had to be modified and extended. A 12-camera Vicon T-Series $(6 \times$ T160, $6 \times$ T40) optical motion capture system was used to capture the data, sampling at $100 \mathrm{~Hz}$.

\section{A. Static Reference Frames}

The modified kinematic measurement technique was validated using a series of precision-made rigs, developed specifically to ascertain the accuracy of the modified kinematic measurement when calculating extreme joint range of motion, 


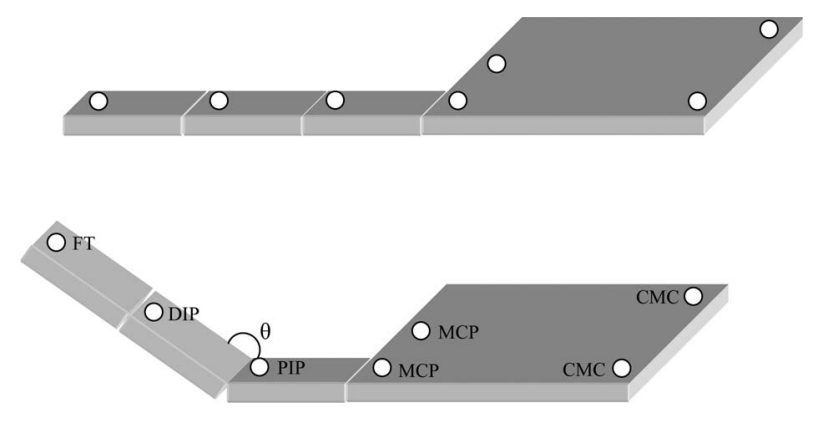

Fig. 3. Precision-made rigs to replicate the movement of MCP, PIP, and DIP joint hyperextension.

as well as to quantify the effect any secondary out-of-plane movement has on the primary measurement. This is particularly relevant when measuring joints that may be misaligned due to pathology. The marker placement as defined in [1] was simulated on surfaces with known angles, shown in Fig. 3. Each component of the rigs was fixed to a position of neutral, $30^{\circ}$ and $60^{\circ}$ flexion and hyperextension, which are ranges including and exceeding those expected of hypermobile joints. The accuracy of the method was defined as the absolute residual error between the target angle, defined by the frames, and the output from the kinematic technique.

\section{B. Dynamic Participant Trial}

The method was applied to existing kinematic data from a patient sample. In the participant trial, as with the previous validation procedure [1], a 6-camera Vicon 460 was used to capture the data sampling at $100 \mathrm{~Hz}$. 3-D marker trajectories were generated from a sample of eight, right-handed participants with RA (one male, seven females; mean age $=62.8$ years \pm 12.04 ). The original study was granted ethical approval by the Hampshire and Isle of Wight NHS Ethics Committee (06/Q1701/139). In order to provide concurrency with the previous study, the heavyweight power object was used from the Southampton hand assessment procedure (SHAP). The power object task mimics the action of an individual reaching and grasping a coffee cup. The power object was chosen as the reaching phase of the movement required the participant to stretch their fingers to maximize potential grip aperture, thus optimizing the potential for finger joint hyperextension to occur.

To complete the power task, the participant was asked to sit in front of a table with their hands resting on the surface either side of the SHAP form-board, which is placed on the table directly in front of them. When instructed to start, the participant pressed the SHAP timer unit, indicating the start of the movement cycle, grasped the power object, placed the object at a specific location on the SHAP form-board, and then pressed the timer unit again. Each participant was assessed lifting the power object five times in succession.

\section{Data Analysis}

Data from the static reference frames and the dynamic participant trials were filtered using the Woltring filter (mean square error value $=10$ ) available in the Vicon Nexus software. The dynamic angles of each joint were calculated using the method described in the next section. Joint angle flexion is represented by a positive angle $(+\mathrm{ve})$ and hyperextension by a negative angle (-ve). Data were normalized for the participant trial to $100 \%$ of the movement cycle by using the MATLAB (MathWorks, Inc., Natick, MA) resampling algorithm. Each participant completed the SHAP power task five times and descriptive statistics (mean angle, minimum angle, maximum angle, and range of motion) were calculated across movement cycles to assess the differences in movement.

From the original eight participants, one female participant was unable to lift the heavy version of the SHAP power object due to the severity of joint deformation. The decision was made to substitute the heavy version of the SHAP power object with its lightweight counterpart, which was of the same height and diameter as the heavier version, so would not alter the grip posture required to lift the object. Therefore, the waveforms for that participant were included in the descriptive statistics.

\section{Measurement Protocol}

The original method calculated movements of the wrist (flexion/extension, radial/ulnar deviation), the dorsal aspect of the transverse metacarpal arch (flexion/extension), finger (flexion/extension of the MCP, PIP, and DIP joints, abduction/adduction of the MCP), and thumb (flexion/extension, palmar abduction/adduction, rotation through to opposition).

The movement of the metacarpal arch is intrinsic in the positioning of the fingers relative to the thumb and therefore inherent in the calculation of finger joint motion. Fig. 2 illustrates the three planes that denote the dorsal aspect of the transverse metacarpal arch: the radial hand plane (RHP), middle hand plane (MHP), and ulnar hand plane (UHP). These planes are defined by vectors from a virtual marker defined midway between CMC2 (proximal head of the second metacarpal at the CMC joint) and CMC5 (proximal head of the fifth metacarpal at the $\mathrm{CMC}$ joint) and from markers placed on the following landmarks: RHP $=\mathrm{MCP} 2$ and MCP3; $\mathrm{MHP}=\mathrm{MCP} 3$ and MCP4; and $\mathrm{UHP}=\mathrm{MCP} 4$ and MCP5. In the preceding cases, MCP2 to MCP5 denote the distal head of the second to fifth metacarpal, respectively.

Finger movement is then calculated relative to a corresponding plane, whereby the second finger is calculated relative to the RHP, the third and fourth fingers are calculated relative to the MHP, and the fifth finger is calculated relative to the UHP. These definitions are based on the finger movements relative to the curvature of the metacarpal arch, for example, the position of the fingers relative to the palm when the hand is held in opposition.

In the original method [1], each phalanx of the finger was represented by a vector defined between markers placed on the MCP, PIP, DIP, and finger tip of each finger. The angle was calculated between two adjoining finger segments (the proxi$\mathrm{mal} / \mathrm{medial}$ and medial/distal phalanges).

In order to calculate hyperextension of the fingers, markers placed on the fingers and corresponding metacarpal arch plane 
TABLE I

Markers and Planes USEd to Calculate Pathological FINGER MOVEMENT

\begin{tabular}{|c|c|c|}
\hline Finger & Hand Plane (Markers) & Finger Planes (Markers) \\
\hline $2^{\text {nd }}$ & $\begin{array}{l}R H P \\
\text { (VM, MCP2, MCP3) }\end{array}$ & $\begin{array}{l}P I P(\mathrm{MCP} 2, \mathrm{MCP} 3, \mathrm{PIP} 2) \\
D I P(\mathrm{MCP} 2, \mathrm{MCP} 3, \mathrm{PIP} 2, \mathrm{DIP} 2) \\
\text { FT (MCP2, MCP3, DIP2, FT2) }\end{array}$ \\
\hline $3^{\text {rd }}$ & $\begin{array}{l}\text { MHP } \\
\text { (VM, MCP3, MCP4) }\end{array}$ & $\begin{array}{l}\text { PIP (MCP3, MCP4, PIP3) } \\
D I P(\mathrm{MCP} 3, \mathrm{MCP} 4, \mathrm{PIP} 3, \mathrm{DIP} 3) \\
F T(\mathrm{MCP} 3, \mathrm{MCP} 4, \mathrm{DIP} 3, \mathrm{FT} 3)\end{array}$ \\
\hline $4^{\text {th }}$ & $\begin{array}{l}\text { MHP } \\
\text { (VM, MCP3, MCP4) }\end{array}$ & $\begin{array}{l}\text { PIP (MCP3, MCP4, PIP4) } \\
\text { DIP (MCP3, MCP4, PIP4, DIP4) } \\
F T \text { (MCP3, MCP4, DIP4, FT4) }\end{array}$ \\
\hline $5^{\text {th }}$ & $\begin{array}{l}\text { UHP } \\
\text { (VM, MCP4, MCP5) }\end{array}$ & $\begin{array}{l}\text { PIP (MCP4, MCP5, PIP5) } \\
\text { DIP (MCP4, MCP5, PIP5, DIP5) } \\
F T \text { (MCP4, MCP5, DIP5, ,T5) }\end{array}$ \\
\hline Thumb & $\begin{array}{l}\text { THP } \\
\text { (CMC1, CMC2, MCP1, } \\
\text { MCP2) }\end{array}$ & $\begin{array}{l}M C P(\mathrm{MCP} 1, \mathrm{MCP} 2, \mathrm{IP}) \\
I P(\mathrm{MCP} 1, \mathrm{MCP} 2, \mathrm{IP}, \mathrm{FT} 1)\end{array}$ \\
\hline
\end{tabular}

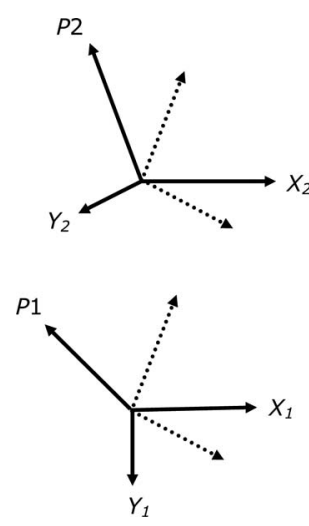

Fig. 4. Coordinate planes of reference and the constituent projected vectors from which out-of-plane movement is considered and calculation of hyperextension generated using (1)-(4).

were used. In addition, a plane was defined for the proximal, medial, and distal phalanges of each finger. Table I describes the markers and planes that are required to calculate joint motion and pathological hyperextension of the MCP, PIP, and DIP joints of each finger. In the general case, let vectors $x_{1}, y_{1}$ and $x_{2}, y_{2}$ lie, respectively, in two planes with $P_{1}, P_{2}$ their respective normal vectors (see Fig. 4). These can then be expressed as

$$
P_{i}=\overline{x_{i} \times y_{i}}, \quad i \in\{1,2\} .
$$

The angular alignment of $P_{2}$ with respect to $P_{1}$ has two components that can be expressed with reference to any pair of orthogonal planes $A_{1}, A_{2}$, each containing $P_{1}$.

The angle of $P_{2}$ with respect to plane $A_{j}$ is

$$
\theta_{j}=\cos ^{-1}\left(\overline{\hat{P}_{2, j} \cdot P_{1}}\right), \quad j \in\{1,2\}
$$

where $\hat{P}_{2, j}$ is the projection of $P_{2}$ onto $A_{j}$, given by

$$
\hat{P}_{2, j}=P_{2} \| A_{j}, \quad j \in\{1,2\} .
$$

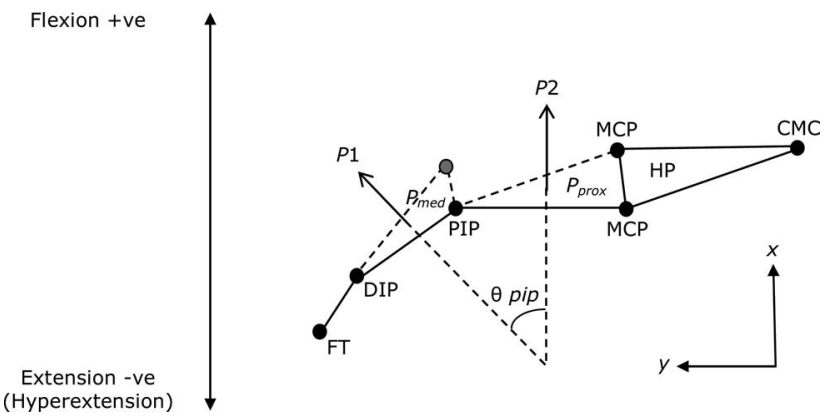

Fig. 5. Depiction of angle calculations from unit vectors normal to the planes of the proximal and medial phalanges of the finger ( $\mathrm{HP}=$ associated hand plane).

To recover the direction of angular alignment, $\theta_{j}$ is multiplied by

$$
\begin{cases}1, & \text { if } \overline{\hat{P}_{2, j} \|\left(P_{1} \times A_{j}\right)}=P_{1} \times A_{j} \\ -1, & \text { otherwise. }\end{cases}
$$

In the specific case considered, $x_{1}=x_{2}$ and the angular alignment is expressed with reference to a single plane with normal vector $A=P_{1} \times y_{1}$. This produces $\hat{P}_{2}=P_{2}$ together with $A \times$ $P_{1}=y_{1}$.

The following example uses the PIP joint of the second finger (index finger); however, it can be extended to any finger or the thumb by substituting the appropriate markers as described in Table I. To calculate PIP joint flexion/extension, a plane was defined from the two vectors to create a proximal phalanx plane, illustrated as $P_{\text {prox }}$ in Fig. 5. A second plane was then defined to create a medial phalanx plane, illustrated as $P_{\text {med }}$ in Fig. 5.

Since vectors have only magnitude and direction, and not position in space, the plane for the medial phalanx of the finger was also defined to move relative to the RHP (or HP in the general sense as depicted in Fig. 5) during flexion and extension by anchoring the $x$-vector defined between the MCP joints (second and third in this case). Unit vectors normal to both planes were then defined using (1). The resultant normal vectors were then projected onto their constituent planes to minimize the effect of secondary out-of-plane movement from any joint, MCP movement or deformity using (2) and (3).

The PIP joint angle was then calculated between the two normal vectors defined for the planes of the proximal and medial phalanges and its direction defined using (4).

When the plane of the medial phalanx passes the point of flexion through to extension (hyperextension in the case of the PIP joint) relative to the plane of the proximal phalanx, the resultant angle would be negative (-ve) and be indicative of pathological movement. Thus, the method described here could provide clinical evidence of PIP joint hyperextension due to joint deformity during dynamic functional activities.

Fig. 5 illustrates the planes as defined by the aforementioned method. During activity, the angle is calculated between the unit vectors normal to the planes $p_{\text {prox }}$ and $p_{\text {med }}$. Fig. 5 depicts the planes and angle calculation using the proposed method. 
TABLE II

RESUlts From Static ReFERENCE FRAMES AND the ModifiEd Kinematic MEASUREMENT TECHNIQUE

\begin{tabular}{ccc}
\hline \hline Reference Angle $^{\circ}$ & Mean Angle $^{\circ}( \pm)$ & Abs. Residual Error $^{\circ}$ \\
\hline-60 & $-58.0(0.7)$ & 2.0 \\
-30 & $-28.4(1.9)$ & 1.6 \\
0 & $-0.2(0.4)$ & 0.2 \\
30 & $26.3(0.2)$ & 3.7 \\
60 & $56.7(2.2)$ & 3.3 \\
\hline \hline
\end{tabular}

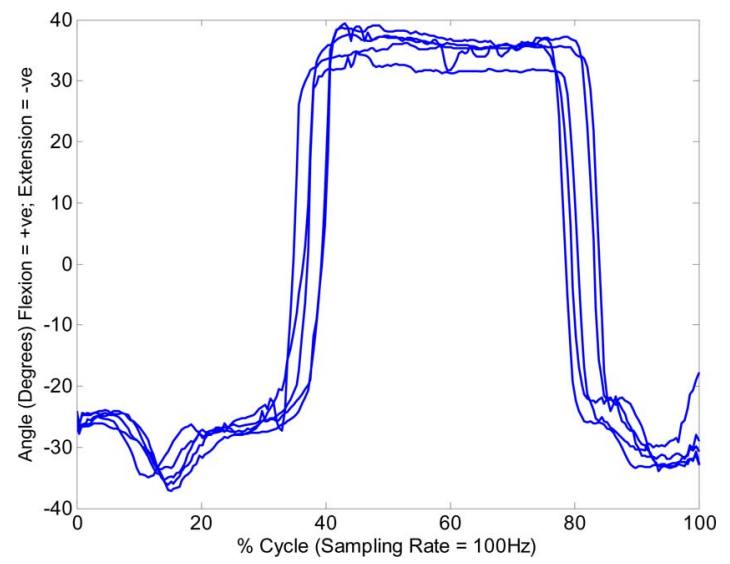

Fig. 6. Sample participant finger joint angles for five repeated movements presented from the PIP joint of the right third (middle) finger.

\section{RESULTS}

\section{A. Static Reference Frames}

Table II summarizes the results of the kinematic measurement technique when applied to the static reference frames.

The results shown in Table II denote the required angle defined by each reference frame, the average angle (MCP, PIP, and DIP joints) from the modified method, and the absolute mean residual, which is the average error of the modified method with respect to the angle of the static reference frame. An absolute residual error of $3.7^{\circ}$ was found between the output from the modified technique and the target angle defined by the frames and a comparison between the angles expected from static reference frames and the modified technique showed a cross correlation of $(r=0.99)$.

\section{B. Dynamic Participant Trial}

The algorithms described here were then applied to previously collected participant data as described in Section III-B. The results show that the majority of participants adopted a similar pattern of movement when asked to repeat the same functional movement as illustrated in Fig. 6.

The most severe occurrence of PIP joint hyperextension was found in the fifth finger of Participant $7\left(-48.8^{\circ}\right)$. However, hyperextension was found not to be present in any other finger of Participant 7 during the repeated lifting task (see Table III). Participant 1 was the only participant not to move into hyperextension at any time during the task.
TABLE III

Descriptive Statistics For the Mean of Five RePEated LifTING Tasks FOR EACH PARTICIPANT

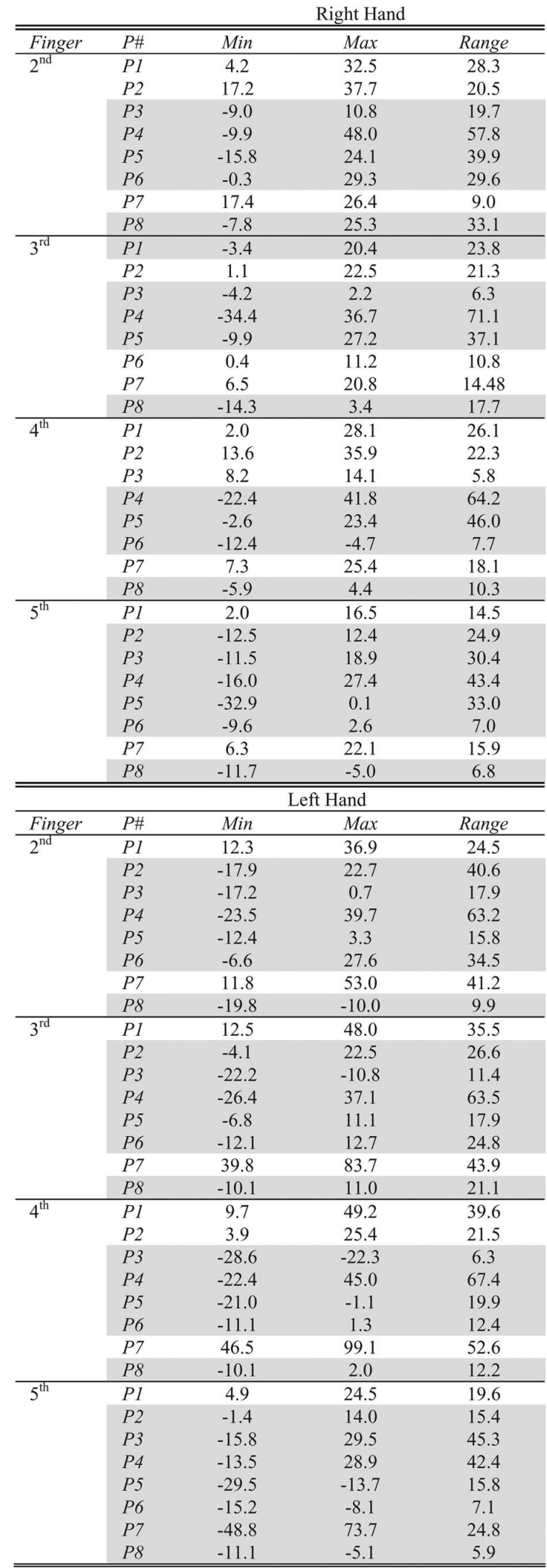

Participants with pathological motion, indicated by hyperextension of the PIP joint are highlighted. 


\section{DISCUSSIONS AND CONCLUSION}

Accurate measurement of finger joint movement is essential to understand the effectiveness of surgical and therapeutic interventions, as well as clinical investigations of a range of neurological and musculoskeletal impairments, such as stroke and arthritic conditions.

The range of motion expected from an unimpaired PIP joint is $0-120^{\circ}$ [10], [11]. Hyperextension of a joint is, therefore, defined as passing through zero (neutral position) and producing a negative resultant angle value.

The modified method presented here has been developed specifically to calculate finger joint movement that exceeds a normative range due to neuromusculoskeletal deformity. The technique has been tested for accuracy using a series of static reference frames and produced an absolute residual error of $3.7^{\circ}$.

Traditional methods, including manual goniometry, are known to be accurate between $7^{\circ}$ and $9^{\circ}$ [24], [25]. To date, the only kinematic method, developed by Chiu et al. [20], developed specifically to calculate finger joint hyperextension generated results for the MCP joint only. In their study, Chiu et al. compared their measurement technique to the TAM, describing a high correlation between the two methods $(r=0.76)$. However, it should be noted that the TAM uses a goniometer to measure static end-of-range movements and is, therefore, susceptible to the measurement errors previously described. The marker placement protocol developed for that method required eight retroreflective markers to calculate MCP, PIP, and DIP joint movement: three placed on the dorsal metacarpal bones of the hand, two placed on both the proximal and medial phalanges, one above the DIP joint, and one on the fingertip.

Other kinematic techniques [14]-[16] are available that would be capable of measuring finger joint hyperextension and have been assessed for accuracy. However, it should be noted that in all cases, the authors do not comment on this application or provide any description that would suggest this was their intention. These techniques may be able to adopt the modified method presented here for that purpose, should it be required in further studies.

Carpinella et al. [26] use a single surface marker protocol and report a repeated accuracy of $\pm 7.3^{\circ}$ and it would be possible to adopt the modified method presented here based on their marker topology. They require five markers to calculate MCP and PIP joint movements, but do not include DIP joint movements. Degeorges et al. [16] use a rod-based series of marker clusters and require 12 markers to calculate MCP, PIP, and DIP joint flexion/extension, and they report an accuracy of $\pm 8.0^{\circ}$. In contrast, the modified method presented here calculates flexion through to hyperextension of all the finger joints and requires seven markers to calculate MCP, PIP, and DIP finger joint movement. In addition, the modified method has been assessed for accuracy using static reference frames for a range between $60^{\circ}$ and $-60^{\circ}$. A comparison between the frames and the modified method show a cross correlation of 0.99 and the results show an absolute residual error of $3.7^{\circ}$, which is notably less than comparable techniques [16], [17], [26].
Due to the limited surface area on the fingers, the method developed by Chiu et al. [20] may have limited application in studying joint movement when deformity is present, particularly relevant when assessing rheumatological impairments. Alternative methods employ a marker cluster topology [15], a technical surface marker set [14] or rigid casts to digitize repeated marker placement between participants [16].

Nussbaum and Zhang [27] discussed the advantages of using a reduced marker set for optical motion capture, and state that increased numbers of markers or complex marker sets can inhibit natural motion. Within the context of assessing functional activities that require dexterous use of multiple fingers, such as grasping objects, methods employing marker cluster topologies [15] or rigid cast systems [16] would, therefore, not be appropriate. However, such methods would be applicable when investigating AROM. Methods that employ more complex surface marker topologies, such as technical marker sets [14], [22], [23] may also prove difficult to apply to patient groups with deformity due to restricted surface available for marker application. The modified method presented here uses a reduced marker set, which is quick and simple to apply, and previously proven to be valid and reliable [1].

The results show various angles of flexion (maximum angle) throughout the majority of the sample, and the angles of extension (minimum angle) do differ considerably, thereby indicating the severity of the swan-neck deformation within the sample (see Table III). Across the sample, seven participants show pathological joint motion into hyperextension throughout the functional task, indicative of swan-neck deformity.

The results also show that an individual will adopt a repeated pattern of movement. This could be due to the restrictions in range of motion placed on the joint associated with the pathology. Previous research has found that unimpaired participants also adopt a repeated pattern of movement when repeating a task many times [21]. This has also been found to be present when assessing neurologically impaired participants following a stroke [21]. The results of the movement strategies adopted by the participants in the present trial, therefore, suggest that the same is true of participants with RA.

The dynamic range of motion of the PIP joint was described by Hume et al. [10] to be between $36^{\circ}$ and $86^{\circ}$ during a functional activity. These measurements were taken from a sample of 35 unimpaired right-handed men aged 26-28 years old.

In contrast, the impaired participants measured in this sample have a range between $5.8^{\circ}$ and $71.1^{\circ}$, thus indicating notably less flexion at the PIP joints during the measured functional activity.

The functional strategies of movement, indicated by the patterns produced by the angle of flexion/extension, were similar between fingers and in right and left hands. However, the range of movement differs for each participant (shown in Table III), which may indicate varying levels of impairment between fingers and participants. These differences are notable in the left, nondominant fingers and are pronounced due to the severity of RA in each participant.

It should be noted that the objective of this study was to measure movement of only the PIP joint and that the influence 
of other joints of the hand affected by the RA were not measured. Therefore, the adjoining joints (MCP and DIP joints) may affect the resultant movement capability of the PIP joint. There is a need to extend the findings of this research to examine the severity of swan-neck deformity in a larger sample to define the range of movement capable for an individual with this deformity. The method proposed in this paper could be used to measure finger joints affected by Boutonnière deformity, which is characterized by flexion of the PIP joint and hyperextension of the DIP joint [2].

The proposed method has successfully been applied to a clinical application showing the effectiveness of splinting techniques for correcting PIP joint hyperextension in participants with RA [6]. The results presented in this paper indicate that by adopting the modified kinematic measurement technique, the deformation of the joint and subsequent hyperextension of the PIP joint can be measured. The differences in negative values shown in Table III describe the severity of the hyperextension at the joint due to swan-neck deformity. The proposed method could be used to assess whether participants with rheumatoid arthritis are hyperextending their fingers during a dynamic functional task, therefore requiring appropriate clinical interventions to minimize and manage the onset and progression of swanneck deformity. The results have shown that dynamic PIP joint hyperextension can be successfully measured using a 3-D motion analysis system and functional range of motion of the PIP joint has been presented for a sample of participants with rheumatoid arthritis.

A method has been defined to calculate finger joint hyperextension based on an existing and validated kinematic measurement technique. This modified technique could be used in clinical research as an alternative way of measuring pathological joint movement in participants with neuromusculoskeletal conditions, and can be used as an extension of the original technique described in [1].

\section{ACKNOWLEDGMENT}

The authors would like to thank C. Macleod, Royal Hampshire County NHS Hospital, Winchester, U.K., for identifying patients, Dr. J. Adams, Faculty of Health Sciences, and C. Spicka, Southampton Universities Hospital Trust, Southampton, U.K., for providing the original participant data in order to validate this theoretical approach and Dr. C. Freeman for his advice regarding notation.

\section{REFERENCES}

[1] C. D. Metcalf, S. V. Notley, P. H. Chappell, J. H. Burridge, and V. T. Yule, "Validation and application of a computational model for wrist and hand movements using surface markers," IEEE Trans. Biomed. Eng., vol. 55, no. 3, pp. 1199-1210, Mar. 2008.

[2] R. Tubinana, J. M. Thomine, and E. Mackin, Examination of the Hand \& Wrist. London: Martin Dunitz, 1996.

[3] S. Ozturk, F. Zor, M. Sengezer, and S. Isik, "Correction of bilateral congenital swan-neck deformity by use of Mitek Mini Anchor: A new technique," Brit. J. Plastic Surg., vol. 58, pp. 822-825, 2005.

[4] M. Carlson, K. Gallagher, and M. Spirtos, "Surgical treatment of swanneck deformity in hemiplegic cerebral palsy," J. Hand Surg., vol. 32, pp. 1418-1422, 2007.
[5] V. Smrčka and I. Dylevsk, "Treatment of congenital swan neck deformity with dynamic tenodesis of the proximal interphalangeal joint," J. Hand Surg., vol. 26B, pp. 165-167, 2001.

[6] J. Adams, C. Macleod, C. D. Metcalf, and C. Spicka, "ARC silver medal award: A research report on the effectiveness of silver ring splints in preventing proximal interphalangeal joint hyperextension during functional activity," J. Rheum.: Occ. Ther, vol. 23, pp. 19-21, 2008.

[7] F. J. Van Der Giesen, W. J. Van Lankveld, C. Kremers-Selten, A. J. Peeters, E. B. Stern, S. Le Cessie, R. G. H. H. Nelissen, and T. P. M. Vliet Vlieland, "Effectiveness of two finger splints for swan neck deformity in patients with rheumatoid arthritis: A randomized, crossover trial," Arth. Rheum, vol. 61, pp. 1025-1031, 2009.

[8] B. S. Ellis and A. Bruton, "A study to compare the reliability of composite finger flexion with goniometry for measurement of range of motion in the hand," Clin. Rehabil., vol. 16, pp. 562-570, 2002.

[9] B. G. Stanley and S. M. Tribuzi, Concepts in Hand Rehabilitation. Philadelphia: F. A. Davis, 1992.

[10] M. C. Hume, H. Gellman, H. McKellop, and R. H. Brumfield, "Functional range of motion of the joints of the hand," J. Hand Surg., vol. 15 A, pp. 240-243, 1990.

[11] L. K. Smith, E. L. Weiss, and L. D. Lehmkuhl, "Mechanical principles: kinematics," in Brunnstrom's Clinical Kinesiology. Philadelphia: F. A. Davis, 1996.

[12] G. Wu, F. C. T. Van Der Helm, H. E. J. Veeger, M. Makhsous, P. van Roy, C. Anglin, J. Nagels, A. R. Karduna, K. McQuade, X. Wang, F. W. Werner, and B. Buchholz, "ISB recommendation on definitions of joint coordinate systems of various joints for the reporting of human motion-part II: Shoulder, elbow, wrist and hand," J. Biomech, vol. 38, pp. 981-992, 2005.

[13] X. Zhang, S. Lee, and P. Braido, "Determining finger segmental centres of rotation in flexion-extension based on surface marker measurement," J. Biomech., vol. 36, pp. 1097-1102, 2003.

[14] N. Miyata, M. Kouchi, T. Kurihara, and M. Mochimaru, "Modelling of human hand link structure from optical motion capture data," in Proc. IEEE/RSJ Int. Conf. Intell. Robot. Sys., Sep./Oct. 2004, vol. 3, pp. 2130 2135.

[15] N. K. Fowler and A. C. Nicol, "Functional and biomechanical assessment of the normal and rheumatoid hand," Clin. Biomech., vol. 16, pp. 660-666, 2001.

[16] R. Degeorges, J. Parasie, D. Mitton, N. Imbert, J.-N. Goubier, and F. Lavaste, "Three-dimensional rotations of human three-joint fingers: An optoelectronic measurement. Preliminary results," Surg. Radiol. Anat., vol. 27, pp. 43-50, 2005.

[17] P. Cerveri, E. De Momi, N. Lopomo, G. Baud-Bovy, R. M. L. Barros, and G. Ferrigno, "Finger kinematic modelling and real-time hand motion estimation," Ann. Biomed., Eng., vol. 35, pp. 1989-2002, 2007.

[18] H.-Y. Chiu, "A method of two-dimensional measurement for evaluating finger motion impairment," J. Hand Surg., vol. 20B, pp. 691-695, 1995.

[19] S. Williams, R. Schmidt, C. Disselhorst-Klug, and G. Rau, "An upper body model for the kinematical analysis of the joint chain of the human arm," J. Biomech., vol. 39, pp. 2419-2429, 2006.

[20] H.-Y. Chiu, S.-C. Lin, F. C. Su, S.-T. Wang, and H.-Y. Hsu, "The use of the motion analysis system for evaluation of loss of movement in the finger," J. Hand Surg., vol. 25B, pp. 195-199, 2000.

[21] C. D. Metcalf. "The relationship between movement and function of the wrist and hand: A clinically focused kinematic study," Ph.D. dissertation, Univ. of Southampton, Southampton, U.K.

[22] F.-C. Su, Y. L. Chou, C. S. Yang, G. T. Lin, and K. N. An, "Movement of finger joints induced by synergistic wrist motion," Clin. Biomech., vol. 20, pp. 491-497, 2005.

[23] H.-Y. Chiu, F. C. Su, S.-T. Wang, and H.-Y Hsu, "The motion analysis system and goniometry of the finger joints," J. Hand Surg., vol. 23B, pp. 788-791, 1998.

[24] B. S. Ellis, A. Bruton, and J. R. Goddard, "Joint angle measurement: A comparative study of the reliability of goniometry and wire tracing for the hand," Clin. Rehab., vol. 11, pp. 314-320, 1997.

[25] B. S. Ellis and A. Bruton, "A study to compare the reliability of composite finger flexion with goniometry for measurement of range of motion in the hand," Clin. Rehab., vol. 16, pp. 562-570, 2002.

[26] I. Carpinella, P. Mazzoleni, M. Rabuffetti, R. Thorsen, and M. Ferrarin, "Experimental protocol for the kinematic analysis of the hand: Definition and repeatability," Gait Posture, vol. 23, pp. 445-454, 2006.

[27] M. A. Nussbaum and X. Zhang, "Heuristics for locating upper extremity joint centres from a reduced set of surface markers," Human Mov. Sci., vol. 19, pp. 797-816, 2000. 


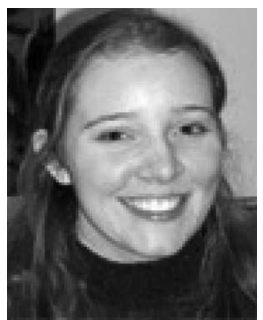

Cheryl D. Metcalf (S'04-M'08) received the B.S. degree (Hons.) in computer studies from the University of Sunderland, Sunderland, U.K., in 2002, specializing in adaptive control strategies of robotic grippers, the M.S. degree in evolutionary and adaptive systems from the University of Sussex, Brighton, U.K., in 2003, specializing in evolving optimized object recognition using haptics, and the Ph.D. degree from the University of Southampton, Southampton, U.K., in 2008, specializing in kinematic analysis of wrist movements and hand function.

She is currently a Research Council's (U.K.) Roberts Fellow (Life Sciences Interface) at the University of Southampton, Southampton, U.K. Her research interests include the hand and wrist biomechanics in unimpaired, neurological and musculoskeletal impairment, developing and assessing novel biomedical sensors for telemonitoring and biomechanical assessment of assistive devices.

Dr. Metcalf is a member of the IEEE Engineering in Medicine, Biology, and Science Society and the International Society of Biomechanics.

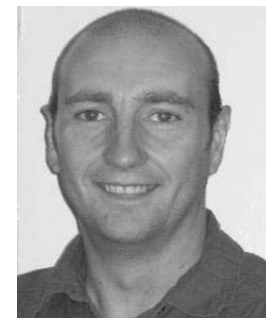

Scott V. Notley received the M.Eng. degree in information engineering and the Ph.D. degree with a dissertation titled "Prediction of Epileptic Seizures from Depth EEG Recordings" from the University of Southampton, Southampton, U.K., in 1995 and 2002, respectively.

$\mathrm{He}$ is currently a Research Fellow at the Institute of Sound and Vibration Research, University of Southampton, Southampton, U.K. His research interests are nonlinear signal processing and biomedical applications of signal processing, particularly applied to electroencephalogram and electromyography. 